\title{
Effect of Planting Density, Irrigation Regimes, and Maize Hybrids with Varying Ear Size on Yield, and Aflatoxin and Fumonisin Contamination Levels
}

\author{
Hamed K. Abbas ${ }^{1 *}$, Henry J. Mascagni Jr. ${ }^{2}$, H. Arnold Bruns ${ }^{3}$, W. Thomas Shier ${ }^{4}$, \\ Kenneth E. Damann ${ }^{5}$ \\ ${ }^{1}$ United States Department of Agriculture-Agricultural Research Service, Biological Control of Pests Research Unit, Stoneville, USA; \\ ${ }^{2}$ Northeast Research Center, Louisiana State University AgCenter, St. Joseph, USA; ${ }^{3}$ United States Department of Agricul- \\ ture-Agricultural Research Service, Crop Production Systems Research Unit, Stoneville, USA; ${ }^{4}$ Department of Medicinal Chemistry, \\ College of Pharmacy, University of Minnesota, Minneapolis, USA; ${ }^{5}$ Department of Plant Pathology \& Crop Physiology, Louisiana \\ State University AgCenter, Baton Rouge, USA. \\ Email: *Hamed.Abbas@ars.usda.gov
}

Received August $31^{\text {st }}, 2012$; revised September $28^{\text {th }}, 2012$; accepted October $5^{\text {th }}, 2012$

\begin{abstract}
Corn (maize, Zea mays L.) hybrids expressing the flexibility trait in ear size (number of kernels per ear) are marketed for ability to give higher yields under adverse conditions. Altered kernel number is associated with altered number of silk, a major route for infection of kernels by aflatoxin-producing fungi such as Aspergillus flavus. The effect of plant density and irrigation level on yield and accumulation of aflatoxins and fumonisins in harvested grain was compared in a fixed-ear hybrid (Pioneer 33K81), a semi-flexible ear hybrid (Pioneer 3223) and a flexible ear hybrid (Golden Acres 8460 ) over a range of seeding densities $\left(49,400,61,750,74,700,86,450\right.$, and 98,800 seeds $\cdot$ ha $\left.^{-1}\right)$ in non-irrigated, moderately-irrigated $(6.4 \mathrm{~cm}$ soil water deficit) and well-irrigated plots $(3.8 \mathrm{~cm}$ soil water deficit), during three years with variable rainfall. Irrigation increased yields in all hybrids, but in the absence of irrigation, yields were highest with the semi-flexible ear trait hybrid. In general, the hybrid with the flexible ear trait had lower optimal seeding densities than the other hybrids for each soil water regime. In general, kernel number was least affected by seeding density in the hybrid with fixed-ear trait compared to the semi- and flexible ear hybrids. The lowest levels of aflatoxin and of fumonisin contamination in harvested grain were associated with the flexible ear trait at all rainfall and irrigation levels, but there was no evidence that reducing stress by lowering seeding density reduced mycotoxin contamination. Inoculation with $A$. flavus resulted in much higher levels of aflatoxin and significantly higher levels of fumonisin contamination in grain of all hybrids under most conditions of rainfall and irrigation, suggesting that factors that promote $A$. flavus infection can affect production of both mycotoxins.
\end{abstract}

Keywords: Aflatoxin; Fumonisin; Mycotoxin; Corn; Maize; Environmental Manipulation; Irrigation; Flexible Kernel Number Trait

\section{Introduction}

Contamination by aflatoxin is a major determinant of crop quality in corn (maize, Zea mays L.) in the US, particularly in the Southern US, where the hot, dry conditions which favor Aspergillus flavus and other aflatoxin-producing fungi frequently occur [1-7]. Fungi cause a variety of root, stalk and ear rots in corn, all of which cause some loss of yield, but it is aflatoxin produced by Aspergillus flavus contaminating kernels that is responsible for the greatest economic losses [8]. Aspergillus flavus spores infect corn after over-wintering in reservoirs provided by

"Corresponding author. the soil and surface plant debris. The husk on the ear constitutes a major physical barrier to infection of kernels by A. flavus, but between silking (reproductive stage R1) and blacklayer (physiological maturity, reproductive stage R6) [9], the silks provide avenues for A. flavus to enter the ear and infect the kernels. The fungus may also infect the kernels by entering through exposed kernels, i.e. insect damage. Once inside the ear, a fungus may spread laterally and infect many kernels [10]. Contamination of harvested corn kernels by another class of mycotoxins, the fumonisins, is also a problem [1-3,7,11-13]. The fungus that produces fumonisins, Fusarium verticillioides (Sacc.) Nirenberg [syn: Fusarium moniliforme], is 
an endophyte present in the seed, which can infect the developing plant during germination. F. verticillioides is also present in the soil, where it is introduced by decaying corn plant material. F. verticillioides can also infect corn plants from the soil reservoir, but the relative contributions of the two infection sources to fumonisin production in kernels is not well established $[14,15]$.

Fungi that produce mycotoxins are often found in corn kernels at harvest [16-21]. Aflatoxin and fumonisins are responsible for illnesses in humans and animals, and cause deterioration of the product resulting in economic losses to growers [8,22-25]. Thus, control of aflatoxin and fumonisins is a worldwide priority [26-28]. The United States Food and Drug Administration (FDA) has issued "action levels" for mycotoxins in foods and feeds in the US, which mandate the maximum level of aflatoxin at $20 \mathrm{ppb}$ in foods for direct human consumption, but lower levels for some animal feed applications, whereas the action level of fumonisins is set at $2 \mathrm{ppm}$ and is advisory $[23,29,30]$. Corn grain may be contaminated by both mycotoxins, which is expected to increase health risks. This regulatory environment has an important economic impact on the grain industry [1-3,31,32].

Kernel number is an important trait that affects a hybrid's ability to adapt to specific growing conditions. Corn hybrids adapted to the Northern and Eastern US, where growing conditions are almost ideal for corn production [33], usually have determinate or fixed kernel number, resulting in uniform cob length and girth even at high plant densities. In contrast, much of the corn production in the Southern US experiences drought stress at some time during the growing season. This is a particular problem on alluvial clay soils, which are more subject to drought stress due to a relatively shallow rooting zone and physical restrictions limiting plant-available water. Irrigation of corn is not consistently done in the lower Mississippi River Valley, particularly on the more slowly draining high clay soils. Many of the newer corn hybrids used in the Southern US have either the indeterminate (-flex) or partially indeterminate (semi-flex) kernel number trait [34]. Flex-ear hybrids respond to good growing conditions by producing an ear with more kernels than the plant would produce at high plant density. This trait may provide a competitive advantage when plant density is low. Examples of factors reducing plant density are soil surface residues which interfere with germination in no-till agriculture or use of lower seeding densities to minimize yield reduction associated with dry conditions. When a flex-ear corn plant responds to lower plant density by producing more kernels, it also produces a corresponding increased number of silks, which provides additional avenues for infection by A. flavus, as well as the desired increase in pollination potential. In principle, the existence of additional routes for A. flavus infection provides the basis for a hypothesis that a flex-ear hybrid will have increased susceptibility to natural infection by $A$. flavus relative to a fixed ear hybrid under similar conditions. Increased infection by A. flavus should result in increased contamination of harvested kernels with aflatoxins, particularly at lower plant density. Differences in aflatoxin contamination in hybrids with different kernel numbers would not be expected when ears are artificially infected with $A$. flavus by a mechanism that circumvents the silks (e.g., a pin-bar applicator). The effect of silk/kernel number on fumonisin contamination is less predictable, because $F$. verticillioides is present as both endophyte and soil-derived pathogen [14,15].

Aflatoxin levels are believed to be affected by the level of stress in corn plants during the ear filling period after silking [7,35]. Among the types of plant stress believed to affect aflatoxin levels, high minimum (i.e., nighttime) temperatures are believed to be very important. However, other types of plant stress including drought and population density may add to heat stress in enabling A. flavus infection, proliferation, and aflatoxin elaboration $[35,36]$. The ability to alter the number of kernels per ear has been introduced into corn cultivars in climates sub-optimal for corn production based on the rationale that these cultivars can minimize plant stress by altering ear size. If this is true, -flex and semi-flex hybrids may experience reduced aflatoxin contamination levels. A typical infection of corn by A. flavus involves the fungus entering the ear by growing down the silk, then spreading laterally in the cob. The larger the ear, the more silks and thus more potential avenues for infection and a greater likelihood of elevated aflatoxin levels. If this mechanism is important, full- and semi-flex corn hybrids would be expected to have higher aflatoxin levels at low seeding densities. The same plant stress factors are expected to affect levels of fumonisin contamination in a way similar to their affects on aflatoxin levels. However, the fumonisin-producing fungus, Fusarium verticillioides, is an endophyte in corn as well as a soil-derived contaminant, and the relative contributions of soil-derived $F$. verticillioides $v s$ endophyte-derived $F$. verticillioides to production of the fumonisins that contaminate corn kernels under different sets of environmental conditions is poorly understood. This situation makes predicting how fumonisin levels in harvested corn kernels would be affected by additional kernels per ear and correspondingly larger numbers of silks more difficult than predicting effects on aflatoxin levels. A study was undertaken to determine the effects of seeding density and irrigation level on 1) Grain yield; 2) Number of kernels per ear and 3) Aflatoxin and fumonisin contamination in harvested corn kernels in hybrids with differing levels of ear de- 
velopment flexibility trait.

\section{Materials and Methods}

\subsection{Experimental Site Characteristics}

Field experiments were conducted in 2000 on the Louisiana Delta Plantation near Jonesville, LA, on an Alligator clay (very-fine, montmorillonitic, acid, thermic Vertic Haplaquepts) and 2001 and 2003 at the Northeast Research Station near St. Joseph, LA, on a Sharkey clay (very-fine, montmorillonitic, non-acid, thermic Vertic Haplaquepts) to evaluate the influence of seeding density on three hybrids differing in ear developmental traits on yield and aflatoxin and fumonisin accumulation in the grain. The following three soil water regimes were established using furrow irrigation: 1) No irrigation; 2) Moderate irrigation, in which crops were watered when the soil water deficit (SWD) reached $6.4 \mathrm{~cm}$; and 3) Wellirrigated, in which crops were watered when the SWD reached $3.8 \mathrm{~cm}$. The experimental design was a randomized complete block with five replications in 2000, three replications in 2001 and four replications in 2003 for each irrigation level. Timing of irrigations were determined using the Arkansas Irrigation Scheduler model [37,38]. In 2000, the well-irrigated regime was watered on June 2, 9, 14 and 26, and July 3, 7, 12, 18 and 25, and moderately irrigated regime was watered on June 2, 14 and 30, and July 11 and 22. In 2001 well-irrigated plots were watered on May 17 and 29, June 20 and 27, and July 3, 10 and 20, and moderately irrigated plots were watered on June 4 and July 2. In 2003 well-irrigated plots were watered on May 30, June 6, 23, and 30, and July 15, 21 and 29, and moderately irrigated plots were watered on June 3 and 27, and July 17 and 29.

\subsection{Corn Hybrids}

Hybrids were selected based on yield potential and differences in ear developmental traits reported by the seed companies. Hybrids evaluated were Pioneer brand (PB) $33 \mathrm{~K} 81$ (a fixed-ear hybrid with a relative maturity of 112 days), PB 3223 (a semi-flex ear hybrid with a relative maturity of 118 days) and Golden Acres (GA) 8460 (a full-flex ear hybrid with a relative maturity of 120 days). However, a randomized block design was used in the study in which each hybrid was grown in a range of five population densities, which should allow valid comparisons of aflatoxin and fumonisin susceptibility to be made within the population density range for each hybrid. Further study will be required to determine the generality of any conclusions drawn from the study concerning relationships between ear developmental traits and mycotoxin susceptibility.

\subsection{Plant Densities}

Corn hybrids were planted with a plot planter at seeding densities of 49,400,61,750, 74,700, 86,450, and 98,800 seeds $\cdot \mathrm{ha}^{-1}$ on 11 April 2000, 23 March 2001, and 2 April 2003. Mid-silk dates occurred in early June. Plots consisted of four rows spaced $102 \mathrm{~cm}$ apart and $12.2 \mathrm{~m}$ long. Cultural practices for fertility and pest control recommended by the Louisiana State University AgCenter were followed. The two center rows of each plot were machine harvested, and yield reported at $15.5 \%$ grain moisture. Yield components of plant population, kernel weight, and kernels per ear were determined. Plant population was determined by counting the harvestable plants in each plot just before harvest. Mean ears per plant were also determined. Kernel weight was determined by averaging the weight of 100 kernels (g per 100 kernels). Kernels per ear was calculated using grain yield, ears $\cdot \mathrm{ha}^{-1}$, and kernel weight in the following formula: Kernels per ear $=$ Yield/(ears ${ }^{\text {-ha }} \times$ kernel weight).

\subsection{Fungal Inoculation}

In order to identify non-silk-related factors in the susceptibility of hybrids to aflatoxin contamination, pin-bar or needle inoculation of ears [39] was used to by-pass the silk-related parts of the infection process, and thereby produce controls for post-infection differences in aflatoxin between hybrids. Primary ears were inoculated with a suspension of about $1.0 \times 10^{6}$ spores $\cdot \mathrm{ml}^{-1}$ of an aflatoxin-producing strain of Aspergillus flavus, F3W4 (NRRL 30796) about 20 days after anthesis (approximately mid-ear stage of development) using the pin-bar method [40] in 2000. In 2001 and 2003, needle inoculation was used. The needle was inserted under the husk and approximately $1.2 \mathrm{ml}$ of suspension $\left(1.0 \times 10^{6}\right.$ spores $\left.\cdot \mathrm{ml}^{-1}\right)$ injected on each ear. In the case of fumonisins, in which kernel contamination can result from both soil-derived and endophyte $F$. verticillioides, the fungus is typically widespread in corn production areas so that artificial inoculation was not necessary.

\subsection{Mycotoxin Determination}

Aflatoxin and fumonisin were determined in naturallyinfected corn from combine-harvested grain. A. flavusinoculated ears were hand-harvested at approximately $15 \%$ grain moisture, shelled, dried at $50^{\circ} \mathrm{C}$ and thoroughly mixed on a sample splitter. Grain samples were ground using a Romer Mill (Romer Lab, Union, MO), and analyzed for aflatoxin and fumonisin at the USDA/ARS laboratory in Stoneville, MS, using commercial ELISA kits (Neogen Corporation, Lansing, MI) as described previously [1-3]. Samples were analyzed in triplicate for both toxins. 


\subsection{Statistical Analyses}

Statistical analyses were conducted using the Proc Mixed procedure of SAS 9.1 [41] to determine the effects of hybrid type and plant density at each irrigation level in each year, and Microsoft Excel 2010 for correlation analysis and Student's t-test. Fisher's Protected Least Significant Difference (LSD) was used to evaluate treatment differences when the F-test indicated a significance level $(\mathrm{P} \leq 0.05)$.

\section{Results and Discussion}

\subsection{Weather Conditions}

Rainfall and temperatures in the Louisiana Delta Plantation (Jonesville) region and St. Joseph for the three years of the study are reported in Tables 1 and 2. Seasonal average temperatures were higher than the 40-year average for both maximum (daytime) and minimum (nighttime) temperatures for each of the three years studied. No single month was consistently responsible for elevated average temperatures. But in 2000, daily maximum temperatures from 14 July through 17 July and 19 July to 20 July were unusually high, ranging from $38.0^{\circ} \mathrm{C}$ to $39.8^{\circ} \mathrm{C}$ (Table 2). Rainfall varied substantially for the three years (Table 1). In 2000 rainfall was far below average in April and July, but at or above average in May and June, respectively. In 2001 rainfall was below average in April and May, while rainfall for June and July was above or near the long-term average, respectively. In
2003 rainfall for April, May, and July was below the long-term average, while June rainfall was well above normal.

Irrigation frequency was greatest in 2000, reflecting the large rainfall deficit that year (Table 1). Irrigation was triggered in the study by soil water deficit, which reflects the length of time between rainfall events as well as low total rainfall and the evapotranspiration rate, which is incorporated in the scheduling model. As a result, in 2000 there were five irrigations for plots in the moderate irrigation category (when the soil water deficit reached $6.4 \mathrm{~cm}$ ) and nine irrigations for plots in the wellirrigated category (when the soil water deficit reached 3.8 $\mathrm{cm}$ ). In 2001, a moderate drought year, there were two irrigations in the moderate irrigation category and seven irrigations in the well-irrigated category. In 2003, a relatively wet year by total rainfall, but with poor distribution, there were four irrigations in the moderate irrigation category and seven irrigations in the well-irrigated category. Soil water levels measured by Watermark sensors (Irrometer Co., Riverside, CA) confirmed that there was a consistent, expected difference in soil water between non-irrigated, moderately irrigated and well-irrigated plots (data not shown).

\subsection{Effects of Planting Density (Seeding Rate) and Soil Water Deficit Kernel Number}

If the hybrids in this study exhibited the kernel number variation expected on the basis of claims made by the

Table 1. Rainfall for the April through July growing season for the three locations in 2000, 2001 and 2003.

\begin{tabular}{ccccc}
\hline Month & \multicolumn{3}{c}{ Rainfall $(\mathrm{mm} / \mathrm{month})$} \\
\hline April & 2000 & 2001 & 2003 & Long-term average $^{1}$ \\
\cline { 2 - 5 } May & 23 & 53 & 69 & 127 \\
June & 137 & 69 & 107 & 135 \\
July & 114 & 155 & 206 & 99 \\
Total & 19 & 107 & 84 & 104 \\
\hline
\end{tabular}

${ }^{1}$ Forty-year average for St. Joseph.

Table 2. Temperatures for the April through July growing season at the experimental sites in 2000, 2001 and 2003.

\begin{tabular}{|c|c|c|c|c|c|c|c|c|}
\hline \multirow{3}{*}{ Month } & \multicolumn{8}{|c|}{ Average Monthly Temperatures $\left({ }^{\circ} \mathrm{C}\right)$} \\
\hline & \multicolumn{2}{|c|}{2000} & \multicolumn{2}{|c|}{2001} & \multicolumn{2}{|c|}{2003} & \multicolumn{2}{|c|}{ Long-term average $^{2}$} \\
\hline & Min & $\operatorname{Max}$ & Min & Max & Min & Max & Min & $\operatorname{Max}$ \\
\hline April & 12.3 & 24.6 & 14.6 & 26.9 & 12.3 & 26.3 & 12.7 & 25.3 \\
\hline May & 19.6 & 31.9 & 17.4 & 30.8 & 19.0 & 30.2 & 17.4 & 29.3 \\
\hline June & 21.3 & 33.0 & 20.7 & 31.4 & 21.3 & 32.5 & 21.1 & 32.6 \\
\hline July & 22.4 & $36.4^{1}$ & 22.4 & 34.2 & 22.4 & 33.6 & 22.7 & 33.9 \\
\hline Season & 18.9 & 31.5 & 18.8 & 30.8 & 18.8 & 30.6 & 18.5 & 30.3 \\
\hline
\end{tabular}

${ }^{1}$ Temperatures for 6 days in mid-July ranged from $38.0^{\circ} \mathrm{C}-39.8^{\circ} \mathrm{C}$. ${ }^{2}$ Forty-year average for St. Joseph. 
marketing seed companies, then the number of kernels per ear would be expected to be affected by environmental factors that stress plants, including plant density and drought. A hybrid marketed as "flex" would be expected to yield ears with fewer kernels under stress conditions, whereas a hybrid marketed as "fixed-ear" would be expected to have yield with similar numbers of kernels under stress conditions. A hybrid marketed as "semiflex" would be expected to exhibit intermediate characteristics. In the present study plant density correlated very closely with seeding density under all conditions encountered ( $R$ values were $0.989 \pm 0.004$ ). Hence, in the following discussion the term "seeding density" is used interchangeably with "plant density". In no treatment were there significantly more than one ear per plant. In 2000 , non-irrigated plots showed no consistent effect of seeding density on kernels per ear in the fixed-ear hybrid. In the semi-flex and flex hybrids, kernel number was reduced by an average of 171 and 199 kernels, respectively, over the range from the lowest to the highest seeding density used (Table 3). Similarly in 2001, nonirrigated plots showed no consistent effect of seeding density on kernels per ear in the fixed-ear hybrid, whereas kernels per ear was reduced by 141 kernels from the lowest to the highest seeding density in the semi-flex hybrid, and reduced by 151 kernels in the full-flex hybrid (Table 4). However, in 2003, kernels per ear in non-irrigated plots was progressively reduced by $125-191$ kernels as the seeding density increased for all three hybrids (Table 5); indicating all three hybrids exhibited either a flex response to plant density, or a response to some unmeasured factor.

Table 3. Effect of hybrids with differing kernel number flexibility trait and seeding density on grain yield and yield characteristics in $2000^{1}$

\begin{tabular}{|c|c|c|c|c|c|c|c|c|c|c|c|c|c|}
\hline \multicolumn{2}{|c|}{ Hybrid/Kernel number } & \multicolumn{4}{|c|}{ Non-irrigated } & \multicolumn{4}{|c|}{ Moderate irrigation (6.4 cm SWD) } & \multicolumn{4}{|c|}{ Well-irrigated (3.8 cm SWD) } \\
\hline Flexibility Trait & $\begin{array}{c}\text { Seeding } \\
\text { density } \\
\text { (seeds/ha)( }\end{array}$ & $\begin{array}{c}\text { Grain } \\
\text { yield } \\
\left(10^{6} \mathrm{~g} / \mathrm{ha}\right)\end{array}$ & $\begin{array}{c}\text { Plant } \\
\text { density } \\
\text { (plts/ha) }\end{array}$ & $\begin{array}{l}\text { Kernel } \\
\text { weight } \\
(\mathrm{g} / 100)\end{array}$ & $\begin{array}{l}\text { Ear size } \\
\text { (kernels } \\
\text { per ear) }\end{array}$ & $\begin{array}{c}\text { Grain } \\
\text { yield } \\
\left(10^{6} \mathrm{~g} / \mathrm{ha}\right)\end{array}$ & $\begin{array}{c}\text { Plant } \\
\text { density } \\
\text { (plts/ha) }\end{array}$ & $\begin{array}{l}\text { Kernel } \\
\text { weight } \\
(\mathrm{g} / 100)\end{array}$ & $\begin{array}{l}\text { Ear size } \\
\text { (kernels } \\
\text { per ear) }\end{array}$ & $\begin{array}{c}\text { Grain } \\
\text { yield } \\
\left(10^{6} \mathrm{~g} / \mathrm{ha}\right)\end{array}$ & $\begin{array}{c}\text { Plant } \\
\text { density } \\
\text { (plts/ha) }\end{array}$ & $\begin{array}{l}\text { Kernel } \\
\text { weight } \\
(\mathrm{g} / 100)\end{array}$ & $\begin{array}{l}\text { Ear size } \\
\text { (kernels } \\
\text { per ear) }\end{array}$ \\
\hline PB 33K81 & 49,400 & 4.3 & 43,500 & 22.0 & 464 & 5.0 & 44,260 & 23.3 & 497 & 5.8 & 48,930 & 25.0 & 481 \\
\hline \multirow[t]{4}{*}{ Fixed-ear } & 61,750 & 5.1 & 54,760 & 19.9 & 478 & 5.7 & 58,240 & 23.3 & 423 & 6.7 & 60,560 & 24.2 & 464 \\
\hline & 74,700 & 5.2 & 63,480 & 19.2 & 433 & 6.4 & 69,010 & 21.8 & 435 & 6.8 & 67,460 & 23.1 & 439 \\
\hline & 86,450 & 5.2 & 73,190 & 19.1 & 375 & 6.1 & 74,740 & 21.4 & 388 & 7.8 & 75,630 & 23.0 & 452 \\
\hline & 98,800 & 6.0 & 81,530 & 17.7 & 454 & 5.8 & 83,190 & 20.9 & 341 & 7.6 & 81,340 & 22.7 & 421 \\
\hline Average & & 5.2 & 63,290 & 19.6 & 441 & 5.8 & 65,890 & 22.1 & 417 & 6.9 & 66,780 & 23.6 & 451 \\
\hline PB 3223 & 49,400 & 6.0 & 51,050 & 24.0 & 496 & 6.8 & 50,760 & 28.8 & 474 & 7.2 & 54,270 & 31.7 & 417 \\
\hline \multirow[t]{4}{*}{ Semi-flex } & 61,750 & 6.2 & 58,140 & 24.4 & 447 & 5.4 & 58,540 & 27.4 & 346 & 7.7 & 63,210 & 30.6 & 403 \\
\hline & 74,700 & 5.7 & 67,280 & 22.4 & 390 & 6.6 & 69,110 & 26.9 & 361 & 8.1 & 70,570 & 29.4 & 399 \\
\hline & 86,450 & 5.5 & 75,430 & 20.9 & 351 & 6.7 & 78,520 & 27.6 & 313 & 7.9 & 80,280 & 28.6 & 343 \\
\hline & 98,800 & 5.8 & 84,840 & 21.5 & 325 & 5.9 & 87,170 & 27.2 & 267 & 8.2 & 91,540 & 27.7 & 328 \\
\hline Average & & 5.8 & 67,350 & 22.6 & 402 & 6.3 & 68,820 & 27.6 & 352 & 7.8 & 71,970 & 29.6 & 378 \\
\hline GA 8460 & 49,400 & 4.6 & 45,320 & 18.2 & 561 & 6.2 & 51,250 & 25.7 & 472 & 6.8 & 54,760 & 27.5 & 458 \\
\hline \multirow[t]{4}{*}{ Full-flex } & 61,750 & 4.8 & 57,950 & 19.2 & 451 & 6.3 & 58,740 & 25.4 & 378 & 6.6 & 60,290 & 25.9 & 430 \\
\hline & 74,700 & 4.7 & 62,320 & 17.1 & 455 & 5.6 & 65,830 & 24.1 & 366 & 7.3 & 72,520 & 24.8 & 406 \\
\hline & 86,450 & 4.5 & 74,740 & 16.4 & 376 & 5.8 & 73,680 & 22.8 & 350 & 7.1 & 76,990 & 24.8 & 375 \\
\hline & 98,800 & 4.6 & 83,580 & 15.6 & 362 & 5.6 & 84,450 & 23.8 & 282 & 6.8 & 86,700 & 24.2 & 325 \\
\hline Average & & 4.6 & 64,780 & 17.3 & 441 & 5.9 & 66,790 & 24.4 & 370 & 6.9 & 70,250 & 25.4 & 399 \\
\hline \multicolumn{14}{|l|}{$\operatorname{LSD}(0.10):$} \\
\hline Ear flexibility (F) & & 0.4 & NS & 0.6 & 19 & 0.4 & NS & 0.6 & 19 & 0.2 & 2,180 & 0.5 & 18 \\
\hline Seeding density (D) & & NS & 3,850 & 0.8 & 24 & NS & 3,850 & 0.8 & 24 & 0.3 & 2,810 & 0.6 & 23 \\
\hline $\mathrm{F} \times \mathrm{D}$ & & 0.8 & $\mathrm{NS}^{1}$ & NS & NS & 0.8 & NS & NS & NS & 0.4 & NS & NS & NS \\
\hline
\end{tabular}

${ }^{1}$ Abbreviations: NS = Non-significant at the 0.10 probability level; LSD = Fisher's least significant difference test; flex $=$ Flexible kernel number trait; SWD = Soil water deficit; plts $=$ Plants. 
Table 4. Effect of hybrids with differing kernel number flexibility trait and seeding density on grain yield and yield characteristics in $2001^{1}$.

\begin{tabular}{|c|c|c|c|c|c|c|c|c|c|c|c|c|c|}
\hline \multicolumn{2}{|c|}{ Hybrid/Kernel number } & \multicolumn{4}{|c|}{ Non-irrigated } & \multicolumn{4}{|c|}{ Moderate irrigation (6.4 cm SWD) } & \multicolumn{4}{|c|}{ Well-irrigated (3.8 cm SWD) } \\
\hline Flexibility Trait & $\begin{array}{c}\text { Seeding } \\
\text { density } \\
\text { (seeds/ha) }\end{array}$ & $\begin{array}{l}\text { Grain } \\
\text { yield } \\
\left(10^{6} \mathrm{~g} / \mathrm{ha}\right)\end{array}$ & $\begin{array}{c}\text { Plant } \\
\text { density } \\
\text { (plts/ha) }\end{array}$ & $\begin{array}{l}\text { Kernel } \\
\text { weight } \\
(\mathrm{g} / 100)\end{array}$ & $\begin{array}{l}\text { Ear size } \\
\text { (kernels } \\
\text { per ear) }\end{array}$ & $\begin{array}{c}\text { Grain } \\
\text { yield } \\
\left(10^{6} \mathrm{~g} / \mathrm{ha}\right)\end{array}$ & $\begin{array}{c}\text { Plant } \\
\text { density } \\
\text { (plts/ha) }\end{array}$ & $\begin{array}{l}\text { Kernel } \\
\text { weight } \\
(\mathrm{g} / 100)\end{array}$ & $\begin{array}{l}\text { Ear size } \\
\text { (kernels } \\
\text { per ear) }\end{array}$ & $\begin{array}{c}\text { Grain } \\
\text { yield } \\
\left(10^{6} \mathrm{~g} / \mathrm{ha}\right)\end{array}$ & $\begin{array}{c}\text { Plant } \\
\text { density } \\
\text { (plts/ha) }\end{array}$ & $\begin{array}{l}\text { Kernel } \\
\text { weight } \\
(\mathrm{g} / 100)\end{array}$ & $\begin{array}{l}\text { Ear size } \\
\text { (kernels } \\
\text { per ear) }\end{array}$ \\
\hline PB 33K81 & 49,400 & 8.0 & 40,780 & 29.7 & 618 & 8.4 & 44,830 & 30.8 & 596 & 8.1 & 42,630 & 31.6 & 597 \\
\hline \multirow[t]{4}{*}{ Fixed-ear } & 61,750 & 9.2 & 55,700 & 30.2 & 549 & 10.1 & 54,170 & 29.9 & 621 & 10.0 & 52,710 & 30.9 & 608 \\
\hline & 74,700 & 9.8 & 57,060 & 28.3 & 606 & 10.9 & 63,530 & 29.5 & 572 & 11.9 & 61,870 & 32.3 & 583 \\
\hline & 86,450 & 10.7 & 55,200 & 28.5 & 678 & 11.6 & 73,580 & 28.0 & 556 & 12.0 & 70,400 & 28.6 & 584 \\
\hline & 98,800 & 11.3 & 71,930 & 25.4 & 619 & 11.8 & 77,110 & 26.3 & 586 & 12.3 & 74,350 & 26.9 & 607 \\
\hline Average & & 9.8 & 56,130 & 28.4 & 614 & 10.6 & 62,640 & 28.9 & 586 & 10.9 & 60,390 & 30.1 & 596 \\
\hline PB 3223 & 49,400 & 9.5 & 39,870 & 35.6 & 617 & 9.9 & 48,040 & 35.4 & 562 & 9.7 & 46,040 & 33.4 & 629 \\
\hline \multirow[t]{4}{*}{ Semi-flex } & 61,750 & 10.6 & 52,390 & 34.2 & 578 & 11.2 & 55,110 & 34.3 & 571 & 11.3 & 55,450 & 36.4 & 549 \\
\hline & 74,700 & 11.5 & 58,930 & 35.8 & 534 & 11.5 & 59,600 & 35.2 & 547 & 11.4 & 58,860 & 33.8 & 585 \\
\hline & 86,450 & 11.3 & 63,840 & 33.7 & 508 & 13.0 & 77,010 & 34.2 & 485 & 13.2 & 71,460 & 35.1 & 517 \\
\hline & 98,800 & 11.8 & 75,530 & 32.9 & 476 & 13.2 & 81,830 & 35.4 & 454 & 13.0 & 79,410 & 32.8 & 500 \\
\hline Average & & 10.9 & 58,110 & 34.4 & 543 & 11.8 & 64,320 & 34.9 & 524 & 11.7 & 62,240 & 34.3 & 556 \\
\hline GA 8460 & 49,400 & 9.7 & 45,990 & 30.0 & 703 & 10.5 & 50,390 & 29.8 & 680 & 10.5 & 50,460 & 30.6 & 673 \\
\hline \multirow[t]{4}{*}{ Full-flex } & 61,750 & 10.7 & 54,880 & 28.5 & 679 & 11.5 & 60,560 & 28.7 & 690 & 11.1 & 57,080 & 29.5 & 653 \\
\hline & 74,700 & 11.4 & 64,910 & 26.7 & 646 & 12.2 & 68,860 & 28.2 & 622 & 12.1 & 69,010 & 32.5 & 553 \\
\hline & 86,450 & 11.0 & 72,450 & 26.3 & 575 & 12.1 & 75,710 & 25.9 & 610 & 11.7 & 74,670 & 27.4 & 568 \\
\hline & 98,800 & 11.2 & 80,600 & 25.2 & 552 & 12.1 & 81,490 & 27.1 & 550 & 11.5 & 78,180 & 27.9 & 527 \\
\hline Average & & 10.8 & 63,770 & 27.3 & 631 & 11.7 & 67,400 & 27.9 & 630 & 11.4 & 65,880 & 29.6 & 595 \\
\hline $\operatorname{LSD}(0.10):$ & & & & & & & & & & & & & \\
\hline Ear flexibility (F) & & 0.4 & 3,580 & 0.8 & 35 & 0.2 & 1,960 & 0.8 & 25 & 0.5 & 2,710 & 1.8 & 33 \\
\hline Seeding density (D) & & 0.6 & 4,620 & 1.0 & 45 & 0.3 & 2,530 & 1.0 & 32 & 0.5 & 3,500 & 2.3 & 42 \\
\hline $\mathrm{F} \times \mathrm{D}$ & & NS & NS & NS & 78 & 0.6 & NS & 1.7 & NS & 0.9 & NS & NS & NS \\
\hline
\end{tabular}

${ }^{1}$ Abbreviations: NS $=$ Non-significant at the 0.10 probability level; LSD = Fisher's least significant difference test; flex $=$ Flexible kernel number trait; SWD $=$ Soil water deficit; plts $=$ Plants.

Table 5. Effect of hybrids with differing kernel number flexibility trait and seeding density on grain yield and yield characteristics in $2003^{1}$.

\begin{tabular}{|c|c|c|c|c|c|c|c|c|c|c|c|c|c|}
\hline \multicolumn{2}{|c|}{ Hybrid/Kernel Number } & \multicolumn{4}{|c|}{ Non-irrigated } & \multicolumn{4}{|c|}{ Moderate irrigation (6.4 cm SWD) } & \multicolumn{4}{|c|}{ Well irrigated (3.8 cm SWD) } \\
\hline Flexibility Trait & $\begin{array}{c}\text { Seeding } \\
\text { density } \\
\text { (seeds/ha)( }\end{array}$ & $\begin{array}{c}\text { Grain } \\
\text { yield } \\
\left(10^{6} \mathrm{~g} / \mathrm{ha}\right)\end{array}$ & $\begin{array}{c}\text { Plant } \\
\text { density } \\
\text { (plts/ha) }\end{array}$ & $\begin{array}{l}\text { Kernel } \\
\text { weight } \\
(\mathrm{g} / 100)\end{array}$ & $\begin{array}{l}\text { Ear size } \\
\text { (kernels } \\
\text { per ear) }\end{array}$ & $\begin{array}{c}\text { Grain } \\
\text { Grain } \\
\left(10^{6} \mathrm{~g} / \mathrm{ha}\right)\end{array}$ & $\begin{array}{c}\text { Plant } \\
\text { density } \\
\text { (plts/ha) }\end{array}$ & $\begin{array}{l}\text { Kernel } \\
\text { weight } \\
(\mathrm{g} / 100)\end{array}$ & $\begin{array}{l}\text { Ear size } \\
\text { (kernels } \\
\text { per ear) }\end{array}$ & $\begin{array}{c}\text { Grain } \\
\text { yield } \\
\left(10^{6} \mathrm{~g} / \mathrm{ha}\right)\end{array}$ & $\begin{array}{c}\text { Plant } \\
\text { density } \\
\text { (plts/ha) }\end{array}$ & $\begin{array}{l}\text { Kernel } \\
\text { weight } \\
(g / 100)\end{array}$ & $\begin{array}{l}\text { Ear size } \\
\text { (kernels } \\
\text { per ear) }\end{array}$ \\
\hline PB 33K81 & 49,400 & 6.9 & 48,580 & 24.8 & 577 & 6.5 & 49,420 & 26.2 & 507 & 7.0 & 45,320 & 27.4 & 570 \\
\hline \multirow[t]{4}{*}{ Fixed-ear } & 61,750 & 7.6 & 60,790 & 23.6 & 536 & 7.2 & 54,980 & 25.2 & 522 & 8.0 & 57,380 & 26.3 & 530 \\
\hline & 74,700 & 8.3 & 67,060 & 23.2 & 536 & 8.4 & 66,440 & 25.6 & 495 & 9.2 & 63,850 & 26.2 & 547 \\
\hline & 86,450 & 8.8 & 81,260 & 22.3 & 485 & 9.7 & 76,770 & 24.1 & 487 & 10.6 & 79,510 & 25.3 & 530 \\
\hline & 98,800 & 8.4 & 89,240 & 22.4 & 424 & 9.5 & 85,220 & 23.3 & 478 & 10.3 & 83,440 & 23.9 & 518 \\
\hline Average & & 8.0 & 69,390 & 23.3 & 512 & 8.3 & 66,570 & 24.9 & 498 & 9.0 & 65,900 & 25.8 & 539 \\
\hline PB 3223 & 49,400 & 8.8 & 47,550 & 31.3 & 595 & 9.5 & 46,360 & 32.7 & 626 & 10.3 & 46,660 & 33.2 & 669 \\
\hline \multirow[t]{4}{*}{ Semi-flex } & 61,750 & 9.6 & 56,070 & 31.2 & 549 & 10.1 & 59,060 & 32.1 & 532 & 11.1 & 57,970 & 33.6 & 573 \\
\hline & 74,700 & 9.2 & 68,740 & 30.0 & 448 & 10.5 & 68,620 & 30.4 & 506 & 11.7 & 69,750 & 31.8 & 527 \\
\hline & 86,450 & 9.9 & 77,360 & 29.9 & 427 & 11.0 & 79,340 & 31.4 & 445 & 12.2 & 75,210 & 32.1 & 508 \\
\hline & 98,800 & 10.3 & 86,850 & 29.3 & 404 & 11.1 & 87,240 & 31.1 & 409 & 12.1 & 82,790 & 32.2 & 456 \\
\hline Average & & 9.6 & 67,310 & 30.3 & 485 & 10.4 & 68,120 & 31.5 & 504 & 11.5 & 66,480 & 32.6 & 547 \\
\hline GA 8460 & 49,400 & 7.8 & 42,310 & 28.2 & 658 & 8.6 & 48,840 & 27.6 & 713 & 9.0 & 43,250 & 29.1 & 722 \\
\hline \multirow[t]{4}{*}{ Full-flex } & 61,750 & 8.6 & 51,670 & 26.7 & 626 & 9.6 & 51,850 & 27.4 & 676 & 10.2 & 52,610 & 28.6 & 681 \\
\hline & 74,700 & 9.4 & 57,670 & 26.0 & 625 & 9.9 & 61,820 & 26.7 & 604 & 10.5 & 60,560 & 27.9 & 624 \\
\hline & 86,450 & 8.7 & 67,280 & 25.4 & 517 & 10.2 & 72,620 & 26.0 & 544 & 11.2 & 68,300 & 27.4 & 602 \\
\hline & 98,800 & 9.4 & 73,580 & 23.9 & 533 & 10.3 & 77,310 & 25.0 & 537 & 11.9 & 77,810 & 26.8 & 572 \\
\hline $\begin{array}{c}\text { Average } \\
\operatorname{LSD}(0.10) \text { : }\end{array}$ & & 8.8 & 58,500 & 26.0 & 592 & 9.7 & 62,490 & 26.5 & 615 & 10.6 & 60,510 & 28.0 & 640 \\
\hline Ear flexibility (F) & & 0.3 & 1,850 & 0.6 & 20 & 0.4 & NS & 0.6 & 26 & 0.3 & 2,800 & 0.4 & 22 \\
\hline Seeding density (D) & & 0.4 & 2,370 & 0.8 & 25 & 0.5 & 4,940 & 0.8 & 33 & 0.4 & 3,620 & 0.5 & 28 \\
\hline $\mathrm{F} \times \mathrm{D}$ & & NS & 4,120 & NS & 44 & NS & NS & NS & 57 & 0.7 & NS & 0.9 & 48 \\
\hline
\end{tabular}

${ }^{1}$ Abbreviations: NS $=$ Non-significant at the 0.10 probability level; LSD $=$ Fisher's least significant difference test; flex $=$ Flexible kernel number trait; SWD $=$ Soil water deficit; plts $=$ Plants. 
The effect of drought on kernel number was less clear. In a comparison of average kernel number in non-irrigated plants between a drought year (2000) and a normal rainfall year (2003), all three hybrids had a lower kernels per ear under drought conditions. But the increase in kernels per ear in the normal rainfall year for the fixed ear hybrid (71 kernels) was less than for the semi-flex hybrid ( 83 kernels), and the largest increase was in the full-flex hybrid (151 kernels). However, the 2001 growing year was anomalous with a larger kernel number for all three hybrids, suggesting another unidentified factor was the primary determinant of kernel number that year. Inconsistent results were also obtained in a comparison of kernels per ear with increasing irrigation levels for the three growing years. Hybrids in the drought year (2000) would be expected to exhibit the largest kernel number flex response. As expected, the fixed ear hybrid changed kernel number little with increased irrigation, but the semi-flex and full-flex hybrids changed kernels per ear in unexpected directions, suggesting that the irrigation levels used were not sufficient to eliminate drought stress. In the semi-drought year (2001) there was insufficient kernels per ear change to allow conclusions to be drawn. In the normal rainfall year (2003) the fixed ear hybrid changed kernels per ear little with increased irrigation, as expected, while the semi-flex and full-flex hybrids increased in kernels per ear (by 64 and 74 kernels, respectively) with increasing irrigation levels as expected for effective relief of drought stress. In general, kernel number change in response to drought was only partially consistent with expectations for ear size change properties claimed by the seed companies that marketed the hybrids.

\subsection{Factors Affecting Yield}

\subsubsection{Effect of Type of Hybrid on Yield}

Hybrids with flexible kernel number are marketed for their purported ability to give higher yields under stress conditions than do fixed ear size hybrids, which have been selected for the ability to give high yields under optimal conditions. When not irrigated, only the main effect of hybrid type was significant. Among the three hybrids in this study, under non-irrigated conditions, the semi-flex hybrid gave the highest average grain yield in all three years, resulting in the average yield rank for the hybrids being semi-flex hybrid $>$ fixed ear hybrid $\approx$ full-flex hybrid (Table 3). These data suggest that the full-flex hybrid may not be very tolerant of dry conditions. The semi-flex hybrid had the highest yield among hybrids regardless of the irrigation level. These higher yields for the semi-flex hybrid were primarily due to higher kernel weights regardless of soil water levels. Hybrid and seeding density main effects affected kernels per ear, however, the interaction between hybrid and seeding density was not significant in either of the soil water treatments in 2000 (Table 3). The fixed ear hybrid exhibited less variation in ear size (kernels per ear) with increased seeding density than the semi-flex and full flex hybrids. In scatter plots of kernels per ear vs seeding density the data for the fixed ear hybrid with no irrigation correlated poorly $\left(R^{2}=0.235\right)$ and the slope of the line fitted to the data $=-0.1$ kernels per ear change per $10^{3}$ increase in seeds per ha. The corresponding values for moderate irrigation were $\mathrm{R}^{2}=0.895$ and slope $=-2.8$, and for well irrigated were $\mathrm{R}^{2}=0.832$ and slope $=-1.1$. For the semi-flex hybrid the corresponding values were $\mathrm{R}^{2}=0.984$ and slope $=-3.6$ for no irrigation, $\mathrm{R}^{2}=0.840$ and slope $=-3.6$ for moderate irrigation and $\mathrm{R}^{2}=0.900$ and slope $=-1.9$ for well-irrigated conditions. For the full flex hybrid the corresponding values were $\mathrm{R}^{2}=0.886$ and slope $=-3.8$ for no irrigation, $\mathrm{R}^{2}=0.894$ and slope $=$ -3.3 for moderate irrigation and $\mathrm{R}^{2}=0.975$ and slope $=$ -2.6 for well-irrigated conditions.

\subsubsection{Effect of Seeding Density on Yield}

Yields from hybrids with flexible kernel number are expected to correlate negatively with seeding density, particularly under drought conditions, because these hybrids can increase yields at low seed densities by increasing kernel number. In the drought year of 2000, yields from semi-flex and full flex hybrids correlated negatively with seeding density with no irrigation $(\mathrm{R}=-0.648$ and -0.412 , respectively) and with low irrigation $(\mathrm{R}=-0.128$ and -0.817 , respectively), whereas under well-irrigated conditions the correlations were positive $(\mathrm{R}=0.863$ and 0.295 , respectively) (Table 3). In contrast, yields from the fixed kernel number hybrid were observed to correlate positively with seeding density with no irrigation $(\mathrm{R}$ $=0.919)$, moderate irrigation $(\mathrm{R}=0.612)$ and well-irrigated $(\mathrm{R}=0.929)$. These differences are reflected in a significant hybrid $\times$ seeding density interaction for yield in both moderately- and well-irrigated corn. In non-irrigated plots in 2000, yields for both the semi-flex hybrid and the full-flex hybrid were maximal at about 74,700 seeds $\cdot \mathrm{ha}^{-1}$, whereas yields for the fixed ear hybrid continued to increase as seeding density increased. Maximum yield for the semi-flex hybrid occurred at higher seeding densities than the other two hybrids in the wellirrigated plots, while yields for the fixed ear hybrid and the semi-flex hybrid increased with higher seeding densities in the moderately irrigated plots.

In years 2001 and 2003, when there was greater rainfall, yields from all hybrids correlated positively with 
seeding density with or without irrigation ( $\mathrm{R}$ ranging from 0.722 to 0.984 ). Kernels per ear for the fixed ear hybrid remained relatively constant across seeding densities, while kernels per ear decreased with increasing plant populations for both the semi-flex hybrid and the fullflex hybrid. In 2001 the main effects of hybrid and seeding density were significant for kernels $\cdot \mathrm{ear}^{-1}$ regardless of soil water level, and the interaction between hybrid and seeding density was significant for the non-irrigated plots. In 2003 there appeared to be a good relationship between optimum plant population and yield potential. Optimum seeding density was approximately 74,700 seed $\cdot \mathrm{ha}^{-1}$ for non-irrigated plots and 86,450 seed $\cdot \mathrm{ha}^{-1}$ for moderately irrigated plots regardless of hybrid. However, there was a significant hybrid $\times$ seeding density interacttion for yield in well-irrigated plots. The optimum seeding density was 86,450 seed $\cdot \mathrm{ha}^{-1}$ for the fixed ear hybrid and 74,700 seed $\cdot \mathrm{ha}^{-1}$ for the semi-flex hybrid, while yield for the full-flex hybrid continued to increase with increased seeding density over the entire range studied. The semi-flex hybrid had the highest kernel weight in non-irrigated, moderately irrigated and well-irrigated plots (Table 5). Unlike the first two years, the hybrid $\times$ seeding density interaction for kernels $\cdot$ ear $^{-1}$ was significant across all irrigation rates examined. The decrease in kernels per ear as plant population increased was less for the fixed ear hybrid than for the other two hybrids.

In general, the flex hybrid had lower optimum plant populations than the other two hybrids for each soil water regime. This was most obvious in both the non-irrigated and moderately irrigated plots, indicating that lower seeding densities could be used for this hybrid, particularly under drought conditions. However, the 2000 findings indicate that the -flex hybrid may not be as drought tolerant as the other hybrids in the study. For a grower, selecting an adapted drought-tolerant hybrid would be advisable in a dryland cropping system.

\subsubsection{Effect of Moisture Stress on Yield}

Reducing drought conditions by irrigation resulted in increased yields with all hybrids in all years and higher levels of irrigation resulted in higher yields (Tables 3-5). Averaged across the three hybrids and seeding densities, yields were $8.2 \mathrm{Mg} \cdot \mathrm{ha}^{-1}$ for the non-irrigated corn, 8.9 $\mathrm{Mg} \cdot \mathrm{ha}^{-1}$ for moderately irrigated corn, and $9.6 \mathrm{Mg} \cdot \mathrm{ha}^{-1}$ for well-irrigated corn, consistent with water stress being greatest in the non-irrigated plots, and least in well-irrigated plots. The largest increase in yield in response to irrigation was observed with the flex hybrid each year, although the relative sizes of yield increases were variable.

\subsection{Mycotoxin Contamination in Hybrids Expressing Varying Levels of Kernel Number Flexibility Trait}

\subsubsection{Aflatoxin Contamination in Hybrids Expressing Varying Levels of Kernel Number Flexibility Trait}

Aflatoxin levels in uninoculated corn averaged over the three years in the study were $33.5 \pm 12.0 \mu \mathrm{g} \cdot \mathrm{kg}^{-1}$ for non-irrigated plots, $29.3 \pm 11.4 \mu \mathrm{g} \cdot \mathrm{kg}^{-1}$ for moderately irrigated plots and $24.2 \pm 8.5 \mu \mathrm{g} \cdot \mathrm{kg}^{-1}$ for well-irrigated plots (Table 7). The effect of drought on aflatoxin levels can be examined by comparing average aflatoxin levels in corn grown without irrigation in the drought year of 2000 , to corn grown in moderately irrigated plots and in well-irrigated plots. In 2000, corn inoculated with A. flavus, averaged aflatoxin levels of $827 \pm 46 \mu \mathrm{g} \cdot \mathrm{kg}^{-1}$ in non-irrigated plots, $783 \pm 51 \mu \mathrm{g} \cdot \mathrm{kg}^{-1}$ in moderately irrigated plots, and $877 \pm 50 \mu \mathrm{g} \cdot \mathrm{kg}^{-1}$ in well-irrigated plots. The only significant difference with differing irrigation levels was hybrid in both the moderately-irrigated and well-irrigated plots. The hybrid with the flexibile kernel number trait had the lowest aflatoxin levels at each of the irrigation levels. In 2001, average aflatoxin levels in corn inoculated with $A$. flavus were $1,008 \pm 274,660 \pm 212$ and $684 \pm 145$ for the non-irrigated, moderately-irrigated and well-irrigated plots, respectively. At each irrigation level, hybrid was significant for aflatoxin levels with the rank being flex ear $>$ fixed ear $=$ semi-flexible ear hybrid . In 2003 aflatoxin levels in corn inoculated with A. flavus were lower than the other two years, averaging $46.5 \pm$ $12.2 \mu \mathrm{g} \cdot \mathrm{kg}^{-1}$ for non-irrigated plots, $68.1 \pm 16.7 \mu \mathrm{g} \cdot \mathrm{kg}^{-1}$ for moderately irrigated plots, and $22.3 \pm 5.5 \mu \mathrm{g} \cdot \mathrm{kg}^{-1}$ for well-irrigated plots. The only significant effect was hybrid in the non-irrigated plots.

Very limited differences in aflatoxin levels in naturally infected corn (Table 6) or in corn inoculated with A. flavus (Table 7) were observed in hybrids with varying ear flex. In 2001, for moderate irrigation there was a significant hybrid type $\times$ seeding density interaction in which aflatoxin levels increased with increasing seeding density for all hybrids with A. flavus inoculation and for the flex hybrid with natural infection. For naturally-infected fixed ear or semi-flex hybrids there was insufficient aflatoxin contamination to evaluate the effect of seeding density in 2001 (Table 6). In 2003 the hybrid $\times$ seeding density interaction was significant only for the non-irrigated plots. There was considerable variation in aflatoxin levels among seeding densities for all hybrids. Effects of drought stress and seeding density alone do not fully explain the variation in aflatoxin levels, consistent with other factors playing a significant role. 

Aflatoxin and Fumonisin Contamination Levels

Table 6. Effect of hybrids with differing kernel number flexibility trait and seeding density on aflatoxin levels in kernels harvested from corn naturally infected with $A$. flavus while growing at three soil water deficit levels in 2000,2001 , and $2003^{1,2,3}$.

\begin{tabular}{|c|c|c|c|c|c|c|c|c|c|c|c|}
\hline \multirow{2}{*}{\multicolumn{2}{|c|}{ Hybrid/Kernel Number }} & \multicolumn{10}{|c|}{ Average aflatoxin levels $\left(\mu \mathrm{g} \cdot \mathrm{kg}^{-1}\right)$} \\
\hline & & \multicolumn{3}{|c|}{ Irrigation in 2000} & \multicolumn{3}{|c|}{ Irrigation in 2001} & \multicolumn{3}{|c|}{ Irrigation in 2003} & \multirow[t]{2}{*}{ Average } \\
\hline Flexibility Trait & $\begin{array}{l}\text { Seeding density } \\
\text { (seeds/ha) }\end{array}$ & None & Moderate & Well & None & Moderate & Well & None & Moderate & Well & \\
\hline PB 33K81 & 49,400 & 99 & 36 & 19 & 14 & 0 & 0 & 1 & 2 & 13 & 20 \\
\hline \multirow[t]{4}{*}{ Fixed-ear } & 61,750 & 41 & 178 & 30 & 0 & 0 & 0 & 1 & 2 & 30 & 31 \\
\hline & 74,700 & 6 & 64 & 14 & 0 & 0 & 0 & 2 & 1 & 1 & 10 \\
\hline & 86,450 & 289 & 141 & 27 & 0 & 0 & 0 & 1 & 66 & 1 & 58 \\
\hline & 98,800 & 7 & 26 & 114 & 0 & 0 & 0 & 23 & 1 & 1 & 19 \\
\hline Average & & 88 & 89 & 41 & 3 & 0 & 0 & 6 & 14 & 9 & 28 \\
\hline PB 3223 & 49,400 & 34 & 109 & 31 & 0 & 0 & 0 & 62 & 1 & 23 & 29 \\
\hline \multirow[t]{4}{*}{ Semi-flex } & 61,750 & 135 & 25 & 12 & 0 & 0 & 0 & 18 & 2 & 1 & 21 \\
\hline & 74,700 & 41 & 63 & 34 & 0 & 0 & 0 & 1 & 2 & 1 & 16 \\
\hline & 86,450 & 7 & 138 & 7 & 0 & 0 & 0 & 7 & 2 & 1 & 18 \\
\hline & 98,800 & 20 & 42 & 147 & 11 & 0 & 0 & 1 & 1 & 1 & 25 \\
\hline GA 8460 & 49,400 & 92 & 7 & 103 & 55 & 19 & 0 & 29 & 3 & 1 & 34 \\
\hline \multirow[t]{4}{*}{ Full-flex } & 61,750 & 256 & 39 & 6 & 32 & 0 & 9 & 1 & 12 & 14 & 41 \\
\hline & 74,700 & 25 & 23 & 10 & 31 & 26 & 113 & 2 & 2 & 2 & 26 \\
\hline & 86,450 & 9 & 37 & 17 & 43 & 48 & 39 & 8 & 2 & 1 & 23 \\
\hline & 98,800 & 85 & 63 & 83 & 16 & 136 & 180 & 3 & 1 & 1 & 63 \\
\hline Average & & 93 & 34 & 44 & 35 & 46 & 68 & 9 & 4 & 4 & 37 \\
\hline $\operatorname{LSD}(0.10):$ & & & & & & & & & $-{ }^{2}$ & & \\
\hline Ear flexibility (F) & & NS & NS & NS & 21 & 22 & 42 & NS & & NS & \\
\hline Seeding density (D) & & NS & NS & 59 & NS & 29 & NS & NS & & NS & \\
\hline $\mathrm{F} \times \mathrm{D}$ & & NS & NS & NS & NS & 50 & NS & NS & NS & NS & \\
\hline
\end{tabular}

${ }^{1}$ Abbreviations: NS = Non-significant at the 0.10 probability level; LSD = Fish's least significant difference test; flex $=$ Flexible kernel number trait; SWD = Soil water deficit. ${ }^{2}$ Some treatments contained only one replicate. ${ }^{3}$ Moderate irrigation occurred at 6.4 -cm SWD; well irrigated occurred at 3.8-cm SWD.

Table 7. Effect of hybrids with differing kernel number flexibility trait and seeding density on aflatoxin levels in kernels harvested from corn inoculated with $A$. flavus while growing at three soil water deficit levels in 2000, 2001, and 2003 ${ }^{1,2}$.

\begin{tabular}{|c|c|c|c|c|c|c|c|c|c|c|c|}
\hline \multirow{2}{*}{\multicolumn{2}{|c|}{ Hybrid/Kernel Number }} & \multicolumn{10}{|c|}{ Average aflatoxin levels $\left(\mu \mathrm{g} \cdot \mathrm{kg}^{-1}\right)$} \\
\hline & & \multicolumn{3}{|c|}{ Irrigation in 2000} & \multicolumn{3}{|c|}{ Irrigation in 2001} & \multicolumn{3}{|c|}{ Irrigation in 2003} & \multirow[t]{2}{*}{ Average } \\
\hline Flexibility Trait & $\begin{array}{l}\text { Seeding density } \\
\text { (seeds/ha) }\end{array}$ & None & Moderate & Well & None & Moderate & Well & None & Moderate & Well & \\
\hline PB33K81 & 49,400 & 950 & 1,010 & 1,067 & 710 & 115 & 212 & 10 & 77 & 40 & 466 \\
\hline \multirow[t]{4}{*}{ Fixed-ear } & 61,750 & 515 & 956 & 854 & 290 & 175 & 86 & 31 & 82 & 30 & 335 \\
\hline & 74,700 & 779 & 547 & 771 & 50 & 67 & 36 & 34 & 8 & 61 & 261 \\
\hline & 86,450 & 871 & 1,117 & 717 & 134 & 330 & 435 & 54 & 63 & 1 & 414 \\
\hline & 98,800 & 688 & 693 & 673 & 260 & 237 & 363 & 41 & 76 & 11 & 338 \\
\hline Average & & 761 & 865 & 816 & 289 & 185 & 226 & 34 & 61 & 29 & 363 \\
\hline PB3223 & 49,400 & 839 & 921 & 1,053 & 475 & 180 & 89 & 20 & 90 & 50 & 413 \\
\hline \multirow[t]{4}{*}{ Semi-flex } & 61,750 & 1,224 & 1,008 & 945 & 230 & 309 & 477 & 65 & 8 & 5 & 475 \\
\hline & 74,700 & 728 & 937 & 1,249 & 207 & 508 & 689 & 11 & 1 & 15 & 483 \\
\hline & 86,450 & 642 & 605 & 1,087 & 567 & 170 & 221 & 36 & 45 & 1 & 375 \\
\hline & 98,800 & 867 & 849 & 1,036 & 620 & 282 & 678 & 36 & 36 & 47 & 491 \\
\hline Average & & 860 & 864 & 1074 & 420 & 290 & 431 & 34 & 36 & 24 & 447 \\
\hline \multirow[t]{4}{*}{ Full-flex } & 61,750 & 821 & 532 & 738 & 1,148 & 830 & 1,381 & 1 & 11 & 34 & 611 \\
\hline & 74,700 & 1,048 & 628 & 633 & 3,152 & 1,345 & 1,373 & 41 & 181 & 1 & 934 \\
\hline & 86,450 & 636 & 670 & 581 & 1,948 & 3,008 & 1,370 & 105 & 18 & 1 & 926 \\
\hline & 98,800 & 936 & 542 & 906 & 2,458 & 1,855 & 1,218 & 21 & 225 & 1 & 907 \\
\hline Average & & 861 & 620 & 737 & 2315 & 1505 & 1394 & 72 & 107 & 15 & 847 \\
\hline $\operatorname{LSD}(0.10):$ & & & & & & & & & & & \\
\hline Ear flexibility (F) & & NS & 231 & 251 & 679 & 431 & 398 & 29 & NS & NS & \\
\hline Seeding density (D) & & NS & NS & NS & NS & 557 & NS & NS & NS & NS & \\
\hline $\mathrm{F} \times \mathrm{D}$ & & NS & NS & NS & NS & 964 & NS & 65 & NS & NS & \\
\hline
\end{tabular}

${ }^{1}$ Abbreviations: NS $=$ Non-significant at the 0.10 probability level; LSD $=$ Fisher's least significant difference test; flex $=$ Flexible kernel number trait; SWD $=$ Soil water deficit. ${ }^{2}$ Moderate irrigation occurred at 6.4-cm SWD; well irrigated occurred at 3.8-cm SWD. 


\subsubsection{Fumonisin Contamination in Hybrids Expressing Varying Ear Flex}

Fumonisin contamination levels in harvested grain were significantly higher in the full- and semi-flex hybrids than in the fixed kernel number hybrid with (Table 8) or without (Table 9) inoculation with A. flavus, under most (six of nine) experimental conditions of rainfall and irrigation level. In hybrids not inoculated with A. flavus (Table 8), fumonisin levels in harvested grain averaged over the three years were significantly lower (analysis of variance, $\mathrm{P}<0.05)$ for the fixed ear hybrid $(1.32 \pm 0.08$ $\left.\mathrm{mg} \mathrm{kg}{ }^{-1}\right)$ than for the semi-flex hybrid $(2.30 \pm 0.22$ $\left.\mathrm{mg} \cdot \mathrm{kg}^{-1}\right)$ and the flex hybrid $\left(3.02 \pm 0.19 \mathrm{mg} \cdot \mathrm{kg}^{-1}\right)$. In hybrids that were inoculated with A. flavus (Table 9), fumonisin levels in harvested grain averaged over the three years were significantly lower (ANOVA, P $<0.01$ ) for the fixed ear hybrid $\left(1.94 \pm 0.17 \mathrm{mg} \cdot \mathrm{kg}^{-1}\right)$ than for the semi-flex hybrid $\left(2.84 \pm 0.19 \mathrm{mg} \cdot \mathrm{kg}^{-1}\right)$ and the flex hybrid $\left(3.80 \pm 0.10 \mathrm{mg} \cdot \mathrm{kg}^{-1}\right)$. Fumonisin levels in corn grain not inoculated with $A$. flavus averaged over the three years in the study was $1.94 \pm 0.20 \mathrm{mg} \cdot \mathrm{kg}^{-1}$ for non-irrigated plots, $2.18 \pm 0.36 \mathrm{mg} \cdot \mathrm{kg}^{-1}$ for moderately irrigated plots, and $2.54 \pm 0.35 \mathrm{mg} \cdot \mathrm{kg}^{-1}$ for well-irrigated plots (Table 8). Inoculation with A. flavus is not expected to affect fumonisin levels unless the two fungi are mutually antagonistic or synergistic during fungal growth and mycotoxin production. Fumonisin levels in corn grain inoculated with $A$. flavus averaged over the three years in the study were $2.13 \pm 0.19 \mathrm{mg} \cdot \mathrm{kg}^{-1}$ for non-irrigated plots, $3.11 \pm 0.39 \mathrm{mg} \cdot \mathrm{kg}^{-1}$ for moderately irrigated plots, and $3.31 \pm 0.46 \mathrm{mg} \cdot \mathrm{kg}^{-1}$ for well-irrigated plots (Table 9), which was significantly higher $(\mathrm{P}<0.05$, Student's $t$ test) than observed for corn in plots not inoculated with A. flavus.

Fumonisin levels in naturally infected corn (Table 8) were significantly affected by hybrid in 2000 and 2003 with higher fumonisin levels being associated with hybrids expressing increased ear size flexibility. In corn inoculated with A. flavus (Table 9) fumonisin levels were significantly affected by hybrid in 2000 in moderate and well irrigated corn and in 2003 with all irrigation levels, also with higher fumonisin levels being associated with hybrids expressing increased ear size flexibility. Overall average fumonisin levels were significantly higher in hydrids expressing increasing ear size flexibility in naturally infected corn (ANOVA, $\mathrm{P}<0.001$ ) and in corn inoculated with A. flavus (ANOVA, $\mathrm{P}<0.001$ ). No consistent effects of irrigation level or seeding density on fumonisin levels were observed in either naturally infected corn (Table 8) or corn inoculated with A. flavus (Table 9).

Table 8. Effect of hybrids with differing kernel number flexibility trait and seeding density on average fumonisin levels in kernels harvested from corn naturally infected with A. flavus while growing at three soil water deficit levels in 2000, 2001, and $2003^{1,2,3}$.

\begin{tabular}{|c|c|c|c|c|c|c|c|c|c|c|c|}
\hline & & \multicolumn{10}{|c|}{ Average aflatoxin levels $\left(\mu \mathrm{g} \cdot \mathrm{kg}^{-1}\right)$} \\
\hline \multicolumn{2}{|c|}{ Hybrid/Kernel number } & \multicolumn{3}{|c|}{ Irrigation in 2000} & \multicolumn{3}{|c|}{ Irrigation in 2001} & \multicolumn{3}{|c|}{ Irrigation in 2003} & \multirow[t]{2}{*}{ Average } \\
\hline Flexibility Trait & $\begin{array}{l}\text { Seeding density } \\
\text { (seeds/ha) }\end{array}$ & None & Moderate & Well & None & Moderate & Well & None & Moderate & Well & \\
\hline PB33K81 & 49,400 & 0.3 & 1.6 & 2.1 & 1.2 & 2.6 & 0.4 & 1.2 & 0 & 0.8 & 1.1 \\
\hline \multirow[t]{4}{*}{ Fixed-ear } & 61,750 & 0.6 & 0.4 & 1.4 & 1.9 & 3.8 & 0.7 & 0.7 & 0 & 1.3 & 1.2 \\
\hline & 74,700 & 0.3 & 1.8 & 2.7 & 1.1 & 1.7 & 1.1 & 1.9 & 0 & 0.9 & 1.3 \\
\hline & 86,450 & 0.7 & 1.7 & 2.4 & 1.8 & 2.9 & 1.5 & 1.6 & 0.1 & 1.2 & 1.5 \\
\hline & 98,800 & 0.4 & 2.6 & 2.3 & 1.7 & 1.8 & 0.4 & 1.9 & 0.1 & 2 & 1.5 \\
\hline Average & & 0.5 & 1.6 & 2.2 & 1.5 & 2.6 & 0.8 & 1.5 & 0 & 1.2 & 1.3 \\
\hline PB3223 & 49,400 & 0.8 & 3.8 & 4.9 & 1.8 & 1.1 & 0.4 & 2.2 & 0 & 1 & 1.8 \\
\hline \multirow[t]{4}{*}{ Semi-flex } & 61,750 & 1.3 & 2.2 & 5.1 & 1.5 & 1.8 & 0.8 & 1.7 & 0 & 1.4 & 1.8 \\
\hline & 74,700 & 0.9 & 3.3 & 5.5 & 1.9 & 2.1 & 1.3 & 3.8 & 3.9 & 1.1 & 2.6 \\
\hline & 86,450 & 0.7 & 6.3 & 6.7 & 3.2 & 0.9 & 0.5 & 2.3 & 0 & 0.8 & 2.4 \\
\hline & 98,800 & 1.5 & 4.3 & 7 & 4.7 & 1 & 0.5 & 5.5 & 0 & 1.7 & 2.9 \\
\hline Average & & 1 & 4 & 5.8 & 2.6 & 1.4 & 0.7 & 3.1 & 0.8 & 1.2 & 2.3 \\
\hline GA8460 & 49,400 & 0.8 & 6 & 6 & 3.4 & 2.5 & 0.7 & 3.5 & 0.9 & 4 & 3.1 \\
\hline \multirow[t]{4}{*}{ Full-flex } & 61,750 & 1.7 & 6.1 & 6.8 & 2 & 1.1 & 1.2 & 1.4 & 0 & 1.2 & 2.4 \\
\hline & 74,700 & 0.5 & 5.1 & 5.2 & 4 & 3.2 & 2.7 & 2.2 & 0 & 2.4 & 2.8 \\
\hline & 86,450 & 1.4 & 9.2 & 8.3 & 2.4 & 1.4 & 1 & 6.7 & 0 & 1.3 & 3.5 \\
\hline & 98,800 & 1.3 & 4.8 & 9.4 & 2.2 & 3.3 & 1.4 & 2.7 & 2 & 2.8 & 3.3 \\
\hline Average & & 1.1 & 6.2 & 7.1 & 2.8 & 2.3 & 1.4 & 3.3 & 0.6 & 2.3 & 3 \\
\hline $\operatorname{LSD}(0.10):$ & & & & & & & & & & & \\
\hline Ear flexibility (F) & & 0.4 & 1.2 & 1.4 & NS & NS & 0.6 & 1.3 & -2 & 0.7 & \\
\hline Seeding density (D) & & NS & 1.6 & NS & 0.9 & NS & 0.7 & NS & - & NS & \\
\hline $\mathrm{F} \times \mathrm{D}$ & & NS & NS & NS & NS & NS & NS & NS & - & NS & \\
\hline
\end{tabular}

${ }^{1}$ Abbreviations: NS = Non-significant at the 0.10 probability level; LSD $=$ Fisher's least significant difference test; flex $=$ Flexible kernel number trait; SWD $=$ Soil water deficit. ${ }^{2}$ Some treatments contained only one replicate. ${ }^{3}$ Moderate irrigation occurred at 6.4-cm SWD; well irrigated occurred at 3.8-cm SWD. 
Table 9. Effect of hybrids with differing kernel number flexibility trait and seeding density on average fumonisin levels in kernels harvested from corn inoculated with A. flavus while growing at three soil water deficit levels in 2000, 2001, and $2003^{1,2}$.

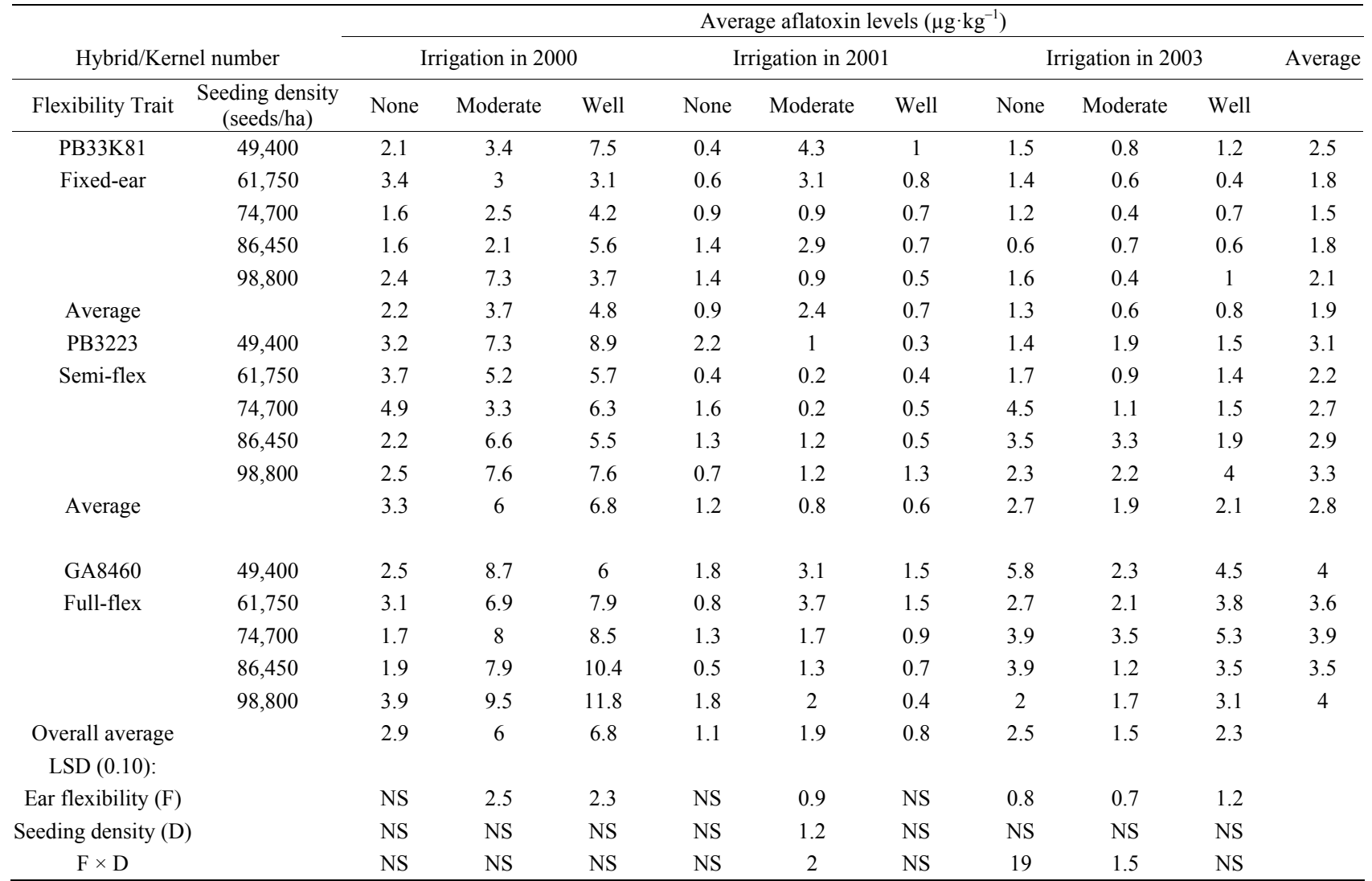

${ }^{1}$ Abbreviations: NS = Non-significant at the 0.10 probability level; LSD = Fisher's least significant difference test; flex $=$ Flexible kernel number trait; SWD = Soil water deficit. ${ }^{2}$ Some treatments contained only one replicate. ${ }^{2}$ Moderate irrigation occurred at 6.4-cm SWD; well irrigated occurred at 3.8-cm SWD.

\subsection{Effect of Irrigation Level on Aflatoxin and Fumonisin Levels}

In the drought year of 2000, aflatoxin levels were significantly higher $(\mathrm{P}<0.001$, Student's $t$-test $)$ than in years with moderate (2001) or good (2003) rainfall at all levels of irrigation with or without inoculation with $A$. flavus (Tables 8 and 9). There were no consistent significant differences in aflatoxin levels among irrigation regimes in the drought year of 2000 or in years with moderate (2001) or good (2003) rainfall, suggesting that factors other than drought were primarily responsible for determining aflatoxin levels in harvested grain.

In the drought year of 2000, fumonisin levels were significantly lower ( $\mathrm{P}<0.001$, Student's $t$-test) in nonirrigated corn than in moderately or well-irrigated corn with or without inoculation with A. flavus (Tables 8 and 9). There were no consistent significant differences in fumonisin levels among irrigation regimes in years with moderate (2001) or good (2003) rainfall, suggesting that factors other than drought were primarily responsible for determining fumonisin levels in harvested grain.

\subsection{Effect of Seeding Rate on Aflatoxin and Fumonisin Levels}

Seeding rate and the resulting plant density is one of several crop management techniques which have been extensively studied for their effects on aflatoxin and fumonisin contamination in harvested corn kernels based on the model that mycotoxin production is associated with factors that increase plant stress [42]. Effects of plant density have become of increased concern as plant population recommendations for maize production have increased to the point at which they are now double those recommended in the 1950's. For example, Rodriguezdel-Bosque [43] observed in Mexico that the two factors most associated with enhanced aflatoxin contamination were late planting and ear insect damage, whereas plant density did not significantly affect aflatoxin contamination. However, Alvarado-Carrillo et al. [44] observed that high plant densities resulted in lower grain yield and increased aflatoxin content and charcoal rot disease in corn in Mexico. In Italy Blandino et al. [45] reported significantly higher levels of fumonisins in harvested corn us- 
ing a combination of agronomic techniques including higher seeding density.

In this study, no consistent significant correlations were observed between seeding density and aflatoxin levels in either naturally infected corn ( $\mathrm{R}$ ranged from -0.73 to +0.71 ) (Table 6) or corn inoculated with A. flavus ( $\mathrm{R}$ ranged from -0.94 to +0.84 ) Table 7). In hybrids not inoculated with A. flavus (Table 8), the average fumonisin levels across the three years correlated well with seeding density for the fixed kernel number hybrid $(\mathrm{R}=$ $0.97)$ and less well for the semi-flexible kernel number hybrid $(\mathrm{R}=0.91)$ and the fully flexible kernel number hybrid $(\mathrm{R}=0.54)$. All significant correlations with seeding density for individual years and irrigation levels in the study were positive, but average fumonisin levels did not correlate $(\mathrm{R}<0.5)$ consistently with seeding density for hybrids ( $\mathrm{R}$ ranged from -0.47 to +0.96 ). In hybrids inoculated with A. flavus (Table 9) there were no consistent significant correlations between fumonisin levels and seeding density ( $\mathrm{R}$ ranged from -0.96 to +0.99 ).

\section{Conclusion}

In this study, characterization of the effects of irrigation and population density (seeding density) on kernel number in corn hybrids with different ear size flexibility traits generally reflected expectations based on seed company claims. Under drought conditions, kernels per ear for the fixed-ear hybrid remained relatively constant across the range of seeding densities studied, whereas kernels per ear decreased with increasing plant population for both the semi-flexible and flexible kernel number hybrids. However, drought alone resulted in smaller numbers of kernels per ear for all hybrids. Irrigation increased yields in all hybrids, but in the absence of irrigation, yields were highest for the hybrid with the semi-flexible kernel number trait. Under irrigation yields of all hybrids correlated with seeding density. Under drought conditions, yields were maximal at 74,700 seeds/ha for semi-flexible and flexible ear hybrids, but yields for the fixed ear hybrid continued to increase with seeding density evaluated. The lowest levels of aflatoxins and fumonisins in harvested grain were observed for the hybrid with the flex ear hybrid at all rainfall and irrigation levels, but there was no evidence that reducing stress by lowering planting density played any role in achieving low mycotoxin contamination. Inoculation with $A$. flavus resulted in much higher levels of aflatoxins and significantly higher levels of fumonisins in all hybrids under most conditions of rainfall and irrigation, suggesting that factors promoting successful infection by $A$. flavus can have a major effect on production of both toxins. Further studies with larger numbers of hybrids will be needed to determine more specific roles played by the ear flex trait

\section{Acknowledgements}

We thank Ms. Bobbie J. Johnson for her technical assistance in this project. Disclaimer: The mention of trade names or commercial products in this report is solely for the purpose of providing specific information and does not imply recommendation or endorsement by the US Department of Agriculture.

\section{REFERENCES}

[1] H. K. Abbas, W. P. Williams, G. L. Windham, J. C. Pringle, W. Xie Jr. and W. T. Shier, "Aflatoxin and Fumonisin Contamination of Commercial Corn (Zea mays) Hybrids in Mississippi," Journal of Agricultural and Food Chemistry, Vol. 50, No. 18, 2002, pp. 5246-5254.

[2] H. K. Abbas, R. D. Cartwright, W. Xie and W. T. Shier, "Aflatoxin and Fumonisin Contamination of Corn (Maize, Zea mays) Hybrids in Arkansas," Crop Protection, Vol. 25, No. 1, 2006, pp. 1-9. doi:10.1016/j.cropro.2005.02.009

[3] H. K. Abbas, W. T. Shier and R. D. Cartwright, "Effects of Temperature, Rainfall and Planting Date on Aflatoxin and Fumonisin Contamination in Commercial Bt and Non-Bt Corn Hybrids in Arkansas," Phytoprotection, Vol. 88, No. 2, 2007, pp. 41-50.

[4] H. A. Bruns and H. K. Abbas, "Responses of Short-Season Corn Hybrids to a Humid Subtropical Environment," Agronomy Journal, Vol. 97, No. 2, 2005, pp. 446-451. doi:10.2134/agronj2005.0446

[5] R. K. Jones, H. E. Duncan and P. B. Hamilton, "Planting Date, Harvest Date, and Irrigation Effects on Infection and Aflatoxin Production by Aspergillus flavus in Field Corn," Phytopathology, Vol. 71, 1981, pp. 810-816. doi:10.1094/Phyto-71-810

[6] E. B. Lillehoj, W. F. Kwolek, A. Manwiller, J. A. Durant, J. C. LaPrade, E. S. Horner, J. Reid and M. S. Zuber, "Aflatoxin Production in Several Corn Hybrids Grown in South Carolina and Florida," Crop Science, Vol. 16, 1976, pp. 483-485.

[7] G. A. Payne, "Aflatoxins in Maize," Critical Review in Plant Science, Vol. 10, No. 5, 1992, pp. 423-440. doi:10.1080/07352689209382320

[8] J. Robens and K. Cardwell, "The Costs of Mycotoxin Management to the USA: Management of Aflatoxins in the United States," Journal of Toxicology-Toxin Reviews, Vol. 22, No. 2-3, 2003, pp. 139-152.

[9] C. S. T. Daughtry, J. C. Cochran and S. E. Hollinger, "Estimating Silking and Maturity Dates of Corn for Large Area," Agronomy Journal, Vol. 76, 1984, pp. 415-420.

[10] H. K. Abbas, J. Wilkinson, R. M. Zablotowicz, C. Accinelli, C. A. Abel, H. A. Bruns and M. A. Weaver, "Ecology of Aspergillus flavus, Regulation of Aflatoxin Production and Management Strategies to Reduce Aflatoxin Contamination of Corn," Toxin Reviews, Vol. 28, No. 2-3, 2009, pp. 142-153. doi:10.1080/15569540903081590

[11] W. J. Chamberlain, C. W. Bacon, W. P. Norred and K. A. 
Voss, "Levels of Fumonisin B1 in Corn Naturally Contaminated with Aflatoxin," Food Chemistry and Toxicology, Vol. 31, No. 12, 1993, pp. 995-998. doi:10.1016/0278-6915(93)90009-N

[12] S. N. Chulze, M. L. Ramirez, M. Farnochi, M. Pascale, A. Visconti and G. March, "Fusarium and Fumonisins Occurrence in Argentinian Corn at Different Ear Maturity Stage," Journal of Agricultural and Food Chemistry, Vol. 44, No. 9, 1996, pp. 2797-2801. doi:10.1021/jf950381d

[13] J. P. Rheeder, W. F. O. Marasas and H. F. Vismer, "Production of Fumonisin Analogs by Fusarium Species," Applied and Environmental Microbiology, Vol. 68, No. 5, 2002, pp. 2101-2105. doi:10.1128/AEM.68.5.2101-2105.2002

[14] C. W. Bacon, A. E. Glenn and I. E. Yates, "Fusarium verticilloides: Managing the Endophytic Association with Maize for Reduced Fumonisins Accumulation," Toxin Reviews, Vol. 27, No. 3-4, 2008, pp. 411-446. doi:10.1080/15569540802497889

[15] G. P. Munkvold, D. C. McGee and W. M. Cariton, "Importance of Different Pathways for Maize Kernel Infection by Fusarium moniliforme," Phytopathology, Vol. 87, No. 2, 1997, pp. 209-217. doi:10.1094/PHYTO.1997.87.2.209

[16] K. F. Cardwell, J. G. King, B. Maziya-Dixon and N. A. Bosque-Perez, "Interactions between Fusarium verticallioides, Aspergillus flavus, and Insect Infestation in Four Maize Genotypes in Lowland Africa," Phytopathology, Vol. 90, No. 3, 2000, pp. 276-284. doi:10.1094/PHYTO.2000.90.3.276

[17] H. K. Abbas, "Special Issue on Aflatoxin and Food Safety, Part II," Journal of Toxicolog-Toxin Reviews, Vol. 23, No. 2-3, 2003, pp. 153-154.

[18] Council for Agriculture Science and Technology, "Mycotoxins: Risks in Plant, Animal, and Human Systems," IA Task Force Report, CAST, Ames, 2003, p. 139

[19] D. C. McGee, O. M. Olanya and G. M. Hoyos, "Populations of Aspergillus flavus in the Iowa Cornfield Ecosystem in Years Not Favorable for Aflatoxin Contamination Corn Grain," Plant Disease, Vol. 80, 1996, pp. 742746. doi:10.1094/PD-80-0742

[20] H. H. L. Gonzalez, S. L. Resnik, R. T. Boca and W. F. O. Marasas, "Mycoflora of Argentinian Corn Harvested in the Main Production Area in 1990," Mycopathologia, Vol. 130, No. 1, 1995, pp. 29-36. doi:10.1007/BF01104346

[21] N. Zummo and G. E. Scott, "Interaction of Fusarium moniliforme and Aspergillus flavus on Kernel Infection and Aflatoxin Contamination in Maize Ears," Plant Disease, Vol. 76, 1992, pp. 771-773. doi:10.1094/PD-76-0771

[22] F. S. Dowling, "Fumonisin and Its Toxic Effects," Cereal Foods World, Vol. 42, No. 1, 1997, pp. 13-15.

[23] National Toxicology Program, "Toxicology and Carcinogenesis Studies on Fumonisin $B_{1}$ in F344/N Rats and B6CF1 Mice (Feed Studies)," Technical Report Series, No. 496. NIH Publication No. 99-3955, US Department of Health and Human Services, National Institutes of Health, Research Triangle Park, 1999.
[24] C. Vardon, C. McLaughlin and C. Nardinelli, "Potential Economic Costs of Mycotoxins in the United States," Council for Agricultural Science and Technology Task Force Report No. 139, Council for Agricultural Science and Technology, Ames, 2003.

[25] N. W. Widstrom, "The Aflatoxin Problem with Corn Grain," Advances in Agronomy, Vol. 56, 1996, pp. 219279. doi:10.1016/S0065-2113(08)60183-2

[26] H. K. Abbas and W. T. Shier, "Mycotoxin Contamination of Agricultural Products in the Southern United States and Approaches to Reducing It from Pre-Harvest to Final Food Products," In: M. Appell, M. Kendra and D. Trucksess, Eds., Mycotoxin Prevention and Control in Agriculture. American Chemical Society Symposium Series, Oxford University Press, Oxford, 2009, pp. 37-58.

[27] H. K. Abbas, R. M. Zablotowicz, B. W. Horn, N. A. Phillips, B. J. Johnson, X. Jin and C. A. Abel, "Comparison of Major Biocontrol Strains of Non-Aflatoxigenic Aspergillus flavus for the Reduction of Aflatoxins and Cyclopiazonic Acid in Maize," Food Additives and Contaminants, Vol. 28, 2011, pp. 198-208. doi: $10.1080 / 19440049.2010 .544680$

[28] J. F. Robens and R. L. Brown, "Aflatoxin/Fumonisin Elimination and Fungal Genomics Workshops," San Antonio, 23-26 October 2002, pp. 393-505.

[29] H. P. van Egmond, R. C. Schothorst and M. A. Jonker, "Regulations Relating to Mycotoxins in Food Perspectives in a Global and European Context," Annals of Bioanalytical Chemistry, Vol. 389, No. 1, 2007, pp. 147-157.

[30] US Food and Drug Administration, "Guidance for Industry: Fumonisin Levels in Human Foods and Animal Feeds," Washington DC, 20 September 2005. http://www. cfsan,fda. gov/ dms/fumongu2. html

[31] J. W. Park, E. K. Kim, D. H. Shon and Y. B. Kim, "Natural Co-Occurrence of Aflatoxin B1, Fumonisin B1 and Ochratoxin A in Barley and Corn Foods from Korea," Food Additives and Contaminants, Vol. 19, No. 11, 2002, pp. 1073-1080. doi:10.1080/02652030210151840

[32] M. Picco, A. Nesci, G. Barros, L. Cavaglieri and M. Etcheverry, "Aflatoxin B1 and Fumonisin B1 in Mixed Cultures of Aspergillus flavus and Fusarium proliferatum on Maize," Natural Toxins, Vol. 7, No. 6, 1999, pp. 331-336.

[33] L. J. Abendroth, R. W. Elmore, M. J. Boyer and S. K. Marlay, "Corn Growth and Development," Iowa State University of Science and Technology Cooperative Extension Service, Ames, 2011.

[34] P. R. Thomison and D. M. Jordan, "Plant Population Effects on Corn Hybrids Differing in Ear Growth Habit and Prolificacy," Journal of Production Agriculture, Vol. 8, No. 3, 1995, pp. 394-400.

[35] K. M. Tubajika, H. J. Mascagni, K. E. Damann and J. S. Russin, "Aflatoxin Production in Corn by Aspergillus flavus Relative to Inoculation, Planting Date, and Harvest Moisture in Louisiana," LSU AgCenter Research Report Number 102, Louisiana State University, Baton Rouge, 2000 .

[36] G. A. Payne, "Process of Contamination by AflatoxinProducing Fungi and Their Impact on Crops," In: K. K. 
Sinha and D. Bhatnagar, Eds., Mycotoxins in Agriculture and Food Safety, Marcel Dekker, New York, 1998, pp. 279-306.

[37] J. Cahoon, J. Ferguson, D. Edwards and P. Tacker, "A Microcomputer-Based Irrigation Scheduler for the Humid Mid-South Region," Applied Engineering in Agriculture, Vol. 6, No. 3, 1990, pp. 289-295.

[38] E. D. Vories, R. Hogan, P. L. Tacker, R. E. Glover and S. W. Lancaster, "Estimating the Impact of Delaying Irrigation for Midsouth Cotton on Clay Soil," Transactions of the American Society of Agricultural and Biological Engineers, Vol. 50, 2007, pp. 929-937.

[39] G. L. Windham, W. P. Williams, P. M. Buckley and H. K. Abbas, "Inoculation Techniques Used to Quantify Aflatoxin Resistance in Corn," Journal of Toxicolog-Toxin Reviews, Vol. 22, No. 2, 2003, pp. 313-325.

[40] H. K. Abbas, R. M. Zablotowicz and H. A. Bruns, "Modeling the Colonization of Maize by Toxigenic and NonToxigenic Aspergillus flavus Strains: Implications for Biological Control," World Mycotoxin Journal, Vol. 1, No.

\section{3, 2008, pp. 333-340. doi:10.3920/WMJ2008.x036}

[41] SAS, "SAS ${ }^{\circledR}$ Proprietary Software Release 9.1," SAS Institute Inc., Cary, 2003.

[42] H. A. Bruns, "Controlling Aflatoxin and Fumonisin in Maize by Crop Management," Journal of Toxicolog-Toxin Reviews, Vol. 22, No. 2, 2003, pp. 153-173.

[43] L. A. Rodriguez-del-Bosque, "Impact of Agronomic Factors on Aflatoxin Contamination in Preharvest Field Corn in Northeastern Mexico," Plant Disease, Vol. 80, 1996, pp. 988-993. doi:10.1094/PD-80-0988

[44] M. Alvarado-Carrillo, A. Diaz-Franco, E. Delgado-Aguirre and N. Montes-Garcia, "Impact of Agronomic Management on Aflatoxin (Aspergillus flavus) Contamination and Charcoal Stalk Rot (Macrophomina phaseolina) Incidence," Tropical and Subtropical Agrosystems, Vol. 12, No. 3, 2010, pp. 575-582.

[45] M. Blandino, A. Reyneri and F. Vanara, "Strategy for Fumonisin Reduction in Maize Kernel in Italy," Mycotoxines fusariennes des Cereals, Arcachon, 11-13 September 2007. http://www.symposcience.org 